\title{
AN EXTENSION OF THE JACOBSON DENSITY THEOREM
}

\author{
BY JULIUS ZELMANOWITZ
}

\author{
Communicated by Robert M. Fossum, January 26, 1976
}

The purpose of this note is to outline a generalization of the Jacobson density theorem and to introduce the associated class of rings.

Throughout $R$ will be an associative ring, not necessarily possessing an identity element. $M_{R}$ will always denote a right $R$-module, and homomorphisms will be written on the side opposite to the scalars. For an element $m \in M$, set $(0: m)=\{r \in R \mid m r=0\}$.

A nontrivial module $M_{R}$ is called compressible if it can be embedded in any of its nonzero submodules. A compressible module $M_{R}$ is critically compressible if it cannot be embedded in any proper factor module. For a compressible module $M_{R}$, each of the following conditions is equivalent to $M_{R}$ being critically compressible: (1) $M_{R}$ is monoform (i.e. nonzero partial endomorphisms of $M$ are monomorphisms); (2) $M_{R}$ is uniform and nonzero endomorphisms of $M$ are monomorphisms. The proof of these characterizations is elementary.

In the absence of more suitable terminology let us define a ring to be weakly primitive if it possesses a faithful critically compressible module. A weakly primitive ring is prime; for it is easy to see that the annihilator of a compressible module is a prime ideal.

In order to simplify this presentation let us call $\left(\Delta,{ }_{\Delta} V_{R}, M_{R}\right)$ an $R$ lattice if $V$ is a $\Delta-R$ bimodule where $\Delta$ is a division ring, $\Delta M=V$, and $R$ acts faithfully on $M$ (so that $R$ can be regarded as a subring of $\operatorname{End}_{\Delta} V$ ). We are now prepared to state the main result.

The Density TheOREM. The following conditions are equivalent for a ring $R$.

(1) $R$ is weakly primitive.

(2) There exists an R-lattice $(\Delta, V, M)$ such that given any elements $m_{1}$, $\ldots, m_{t} \in V$ linearly independent over $\Delta$ there exists $0 \neq a \in \Delta$ such that for any elements $n_{1}, \ldots, n_{t} \in M$ one can find $r \in R$ with $a n_{i}=m_{i} r \in M$ for each $i=1, \ldots, t$.

(3) There exists an R-lattice $(\Delta, V, M)$ such that given any $\tau \in \operatorname{End}_{\Delta} V$

AMS (MOS) subject classifications (1970). Primary 16A20, 16A42, 16A48; Secondary $16 \mathrm{A1} 2,16 \mathrm{A1} 18,16 \mathrm{~A} 64$.

Key words and phrases. Primitive rings, weakly primitive rings, Jacobson density theorem, compressible modules, dense rings of linear transformations.

Copyright $\odot 1976$, American Mathematical Society 
and any elements $m_{1}, \ldots, m_{t} \in M$ linearly independent over $\Delta$ there exist $r$, $s \in R$ with $m_{i} \tau r=m_{i} s$ and $0 \neq m_{i} r \in \Delta m_{i}$ for each $i$.

Let us call a ring $R$ satisfying (2) a weakly dense ring of linear transformations. Observe that weak density is just Jacobson density "up to a scalar"; while condition (3) can be taken to mean that $R$ is a local order in the ring of linear transformations $\operatorname{End}_{\Delta} V$, since $\tau=s r^{-1}$ when restricted to the $\Delta$-subspace generated by $m_{1}, \ldots, m_{t}$. Thus the weakly primitive rings simultaneously embrace primitive rings and orders in simple artinian rings. Many other equivalent variations of these conditions can be given. A density statement similar to, but not quite the same as $(1) \Leftrightarrow(2)$, was given in [4]. Condition (3) is apparently completely new in this setting.

The proof that (1) implies (2) is no more difficult than the proof of the corresponding part of the Jacobson density theorem, once one recognizes the following facts, the first of which is well known.

LEMMA 1. Let $M_{R}$ be a quasi-injective module such that $m R=0$ implies that $m=0$. Then $(0: m) \supseteq \bigcap_{i=1}^{t}\left(0: m_{i}\right)$ if and only if $m=\Sigma_{i=1}^{t} \alpha_{i} m_{i}$ for some $\alpha_{1}, \ldots, \alpha_{t} \in$ End $M_{R}$.

LEMMA 2. If $M_{R}$ is a critically compressible module then End $M_{R}$ is a right Ore domain with quotient division ring End $\bar{M}_{R}$, where $\bar{M}_{R}$ denotes the quasi-injective hull of $M_{R}$.

We mention in passing that End $\bar{M}_{R}$ above is isomorphic to the endomorphism ring of the injective hull of $M_{R}$ modulo its Jacobson radical.

The proof that (2) implies (3) proceeds by induction on $t$, the difficult part being the case $t=1$. Assuming (3) holds, one completes the proof in a straightforward manner by showing that every cyclic submodule of $M_{R}$ is a faithful critically compressible $R$-module. Fine details of the proof will be presented elsewhere.

At this point one is in a position to reproduce the entire theory of primitive rings for the larger class of weakly primitive rings. Here we will be content to state the following consequence which best reveals the nature of these rings, and provides a generalization of the Faith-Utumi theorem [2].

THEOREM 1. If $R$ is a weakly dense subring of $\operatorname{End}_{\Delta} V$, then either $R$ is $a$ right order in a matrix ring of the form $\Delta_{n}$, in which case $R$ contains a subring isomorphic to $D_{n}$ for some right order $D$ in $\Delta$; or else for each positive integer $n$ there exists a homomorphism of a subring of $R$ onto a matrix ring $D_{n}$ for some right order $D$ of the division ring $\Delta$ (the choice of $D$ depends on $n$ ).

Let us next consider the special classes of weakly primitive rings which have been studied in the past.

THEOREM 2. $R$ is a weakly dense ring of linear transformations possess- 
ing a linear transformation of finite rank if and only if $R$ is a prime right nonsingular ring with a uniform right ideal; equivalently, if and only if $R$ has $a$ faithful critically compressible right ideal.

The dense embedding of these rings has been studied extensively in [1]. Next we give a characterization of orders in simple artinian rings which has a representation-theoretic flavor quite different from the famous one given by Goldie [3].

THEOREM 3. $R$ is a right order in a finite matrix ring over some division ring if and only if $R$ has a faithful critically compressible module $M_{R}$ such that $\operatorname{dim}_{\Delta} \Delta M<\infty$, where $\Delta$ is the endomorphism ring of the quasi-injective hull of $M$.

It is perhaps worth mentioning that another equivalent characterization, which arises naturally in the course of proving Theorem 3, is that $R$ be a prime ring which satisfies the finite intersection property (or the d.c.c.) on right annihilators and possesses a uniform right ideal. This was also observed in [5].

An example of a weakly primitive ring not included among the above special cases is $D\{x, y\}$, the free algebra on two generators over a commutative integral domain $D$. This can be verified by minor modification of the argument which shows that the free algebra on two generators over a field is primitive.

\section{BIBLIOGRAPHY}

1. S. A. Amitsur, Rings of quotients and Morita contexts, J. Algebra 17 (1971), 273298.

2. C. Faith and Y. Utumi, On Noetherian prime rings, Trans. Amer. Math. Soc. 114 (1965), 53-60. MR 30 \#3111.

3. A. W. Goldie, Semi-prime rings with maximum condition, Proc. London Math.

Soc. (3) 10 (1960), 201-220. MR 22 \#2627.

4. K. Koh and A. C. Mewborn, A class of prime rings, Canad. Math. Bull. 9 (1966), 63-72. MR 33 \#656; erratum, 34, p. 1785.

5. J. Zelmanowitz, The finite intersection property on annihilator right ideals, Proc. Amer. Math. Soc. (to appear).

DEPARTMENT OF MATHEMATICS, UNIVERSITY OF CALIFORNIA, SANTA BARBARA, CALIFORNIA 93106 